\title{
Oxygen free radicals and congestive heart failure
}

\author{
J J F Belch, A B Bridges, N Scott, M Chopra
}

\begin{abstract}
Plasma lipid peroxides (malondialdehyde) and thiols were measured in 45 patients with congestive heart failure and 45 controls. Malondialdehyde concentrations were significantly higher in the patients with congestive heart failure (median $9.0 \mathrm{nmol} / \mathrm{ml}$ interquartile range (IQR) $7 \cdot 9-10 \cdot 2$ ) than in the controls (median 7.7 $\mathrm{nmol} / \mathrm{ml}$ (IQR 6.9-9.2)). Plasma thiols were significantly lower in congestive heart failure (median 420 $\mu \mathrm{mol} / 1$ (IQR 379-480)) than in the controls (median $463 \mu \mathrm{mol} / 1$ (IQR 445-525)). There was a significant but weak negative correlation between malondialdehyde and left ventricular ejection fraction $(\mathbf{r}=-0.35)$ and a positive correlation between plasma thiols and left ventricular ejection fraction $(r=0.39)$.

This study provides clinical support for experimental data indicating that free radicals may be important in heart failure. It also suggests that the degree of free radical production may be linked to the severity of the disease.
\end{abstract}

In congestive heart failure the heart is unable to provide sufficient blood for tissue metabolic needs. Congestive heart failure affects about $1 \%$ of the population ${ }^{1}$ and carries a poor prognosis with an annual mortality of between $35 \%$ and $58 \% .^{2}$ The pathophysiology of this disorder is undoubtedly multifactorial but the cellular mechanisms at the molecular level have not been fully elucidated.

Oxygen free radicals have been implicated in cardiac ischaemic injury. ${ }^{34}$ These free radicals (superoxide anions and hydroxyl radicals) are produced in the body by reduction of oxygen. In normal circumstances they are removed by the different scavenger systems present in blood and tissue. We believe that free radicals are involved in the genesis of heart failure. In congestive heart failure myocardial contractility is impaired by loss of muscle or by pressure or volume overload. ${ }^{5}$ This pressure and volume overload causes myocardial ischaemia which can generate free radicals. ${ }^{34}$ Support for this hypothesis comes from work in animal models of heart failure where doxorubicin toxicity may result from myocardial injury induced by oxidative damage. ${ }^{6}$ Furthermore, it has been shown that polymorphonuclear leucocytes from dogs with congestive heart failure produce increased amounts of free radicals. ${ }^{7}$ In humans congestive heart failure is associated with an increase in production of prostaglandins. The formation of prostaglandins is associated with the production of oxygen derived free radicals through the arachidonic acid pathway. ${ }^{8}$ Furthermore, circulating catecholamine production increases in the failing heart and their auto-oxidation generates free radicals. ${ }^{9}$ There is therefore some evidence to suggest an involvement of free radicals in congestive heart failure.

We measured the products of free radical reactions in patients with congestive heart failure. The inherent reactivity of radical species with organic biomolecules precludes their direct measurement in biological systems. Their presence has to be inferred from their known effects, such as the induction of protein fluorescence and oxidation of polyunsaturated fatty acids. Two such systems, the measurement of lipid peroxides by the thiobarbituric acid test ${ }^{10}$ and the measure of plasma thiols, ${ }^{11}$ have been carried out here and correlated with the left ventricular ejection fraction-a measure of the severity of congestive heart failure.

\section{Patients and methods}

We studied 45 patients aged under 75 with congestive heart failure diagnosed by a team of hospital physicians. Most patients were thought to have atherosclerosis as the underlying cause of their congestive heart failure. All patients gave informed consent for the study and the study was approved by the hospital ethics committee. Patients with other disorders or receiving medication known to affect free radical activity were excluded from the study. No adjustment was made to the patient's medication, which was taken as usual on the day of the study. Results obtained were compared with 45 age, sex, and smoking matched controls.

The left ventricular ejection fraction was measured in the patients with congestive heart failure as follows: the patient's red cells were labelled in vivo by an injection of stannous pyrophosphate. Twenty minutes later, an injection of $740 \mathrm{MBq}$ of technetium-99m pertechnetate was given. Data were acquired in the anterior and optimised left anterior oblique views with an Ohio Nuclear Series 100 gamma camera linked to a Nodecrest V70 computer. Sixteen $64 \times 64$ images were obtained during each cardiac cycle by an electrocardiographic gating technique. The left ventricular ejection fraction was calculated from the left anterior oblique view; the angle of this was carefully chosen to maximise the
Dr J J F Belch, Department of Medicine, Ninewells Hospital and Medical
School, Dundee DD1 9SY Accepted for publication 17 January 1991 
Table Comparability of groups

\begin{tabular}{lcc}
\hline & Controls & CHF \\
\hline Number & 45 & 45 \\
Age (years) median & 58 & 62 \\
Sex M:F range & $(27-68)$ & $(40-74)$ \\
& $29: 16$ & $37: 8$
\end{tabular}

$\mathrm{CHF}$, congestive heart failure.

separation of the right and left ventricles. A degree of caudal tilt was used to increase the separation of the left atrium from the left ventricle.

Immediately before the left ventricular ejection fraction was measured venous blood samples were collected in a standard manner into a lithium heparin anticoagulant (10 units/ $\mathrm{ml}$ ) tube. The samples were immediately processed and assayed for plasma malondialdehyde-like material. Plasma lipid peroxide concentrations were estimated by Aust's spectrophotometric assay. ${ }^{10}$ Absorbance was converted to concentration by an extinction coefficient of $1.50 \times 10^{5} \mathrm{~mol}^{-1} \mathrm{~cm}^{-1}$. The amount of malondialdehyde-like material is a measure of lipid peroxidation and its measure provides an estimate of free radical activity. Plasma thiol is an index of the oxidative status of the extracellular environment. Plasma thiol was estimated by the method described by Ellman. ${ }^{11}$

\section{Results}

The table shows the comparability of the patients with congestive heart failure and the controls. There was no difference between the two groups $\left(\chi^{2}\right.$ test).

Figure 1 shows the concentration (median (IQR)) of malondialdehyde-like material in both the control population and in patients with congestive heart failure $(7 \cdot 7(6 \cdot 9-9 \cdot 2) v 9$ $(7 \cdot 9-10 \cdot 2) \mathrm{nmol} / \mathrm{ml})$. The patients with congestive heart failure had significantly higher con-

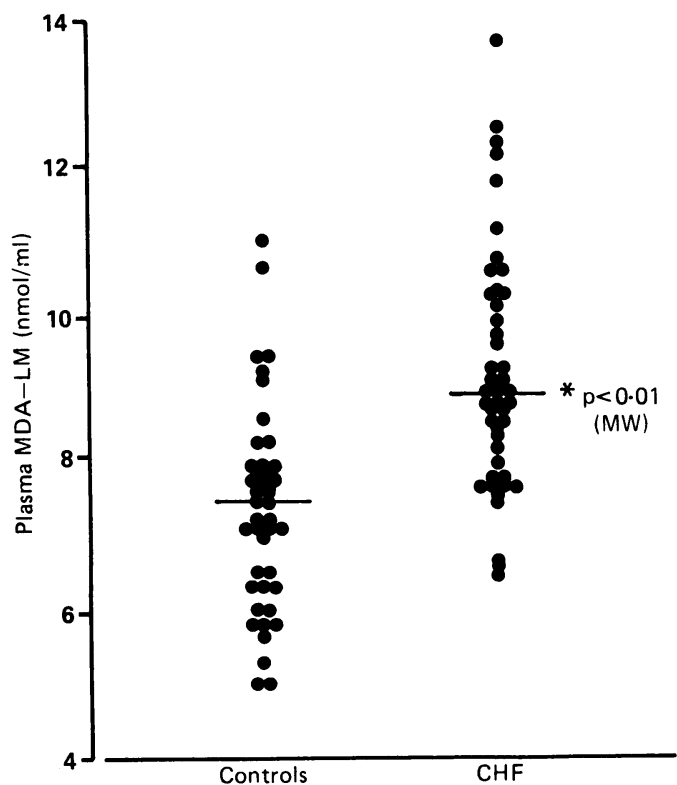

Figure 1 Plasma concentrations of malondialdehydelike material $(M D A-L M)$ in patients with congestive heart failure (CHF) and in controls.

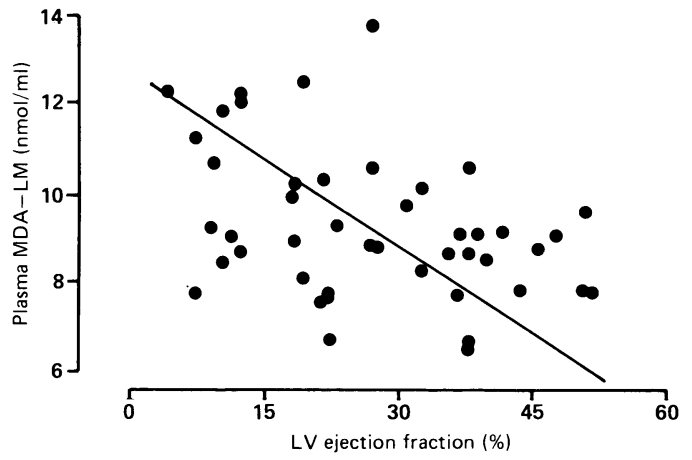

Figure 2 Plasma concentrations of malondialdehydelike material ( $M D A-L M$ ) and left ventricular ( $L V)$ ejection fraction in patients with congestive heart failure.

centrations of malondialdehyde-like material ( $\mathrm{p}<0.01$, Mann-Whitney).

Figure 2 shows the left ventricular ejection fraction of the 45 patients plotted against the concentration of malondialdehyde-like material. There was a statistically significant inverse correlation between the two $(\mathrm{r}=-0.35$, $\mathrm{p}<0.05$, Spearman rank). The higher the concentration of the malondialdehyde-like material the worse the left ventricular ejection fraction.

Figure 3 shows the concentration of plasma thiol (median (IQR)) in both the controls and patients with congestive heart failure (463 $\mu \mathrm{mol} / 1$ (445-525) v 420 (379-480)). The patients with congestive heart disease had significantly lower concentrations of plasma thiol ( $p<0.01$, Mann-Whitney)

Figure 4 shows the relation between left ventricular ejection fraction and plasma thiol concentration. Again there was a statistically significant correlation, this time positive $(\mathrm{r}=0.39, \mathrm{p}<0.01$, Spearman rank); as plasma thiol fell the left ventricular ejection fraction became worse.

\section{Discussion}

We found that there was an increase in lipid

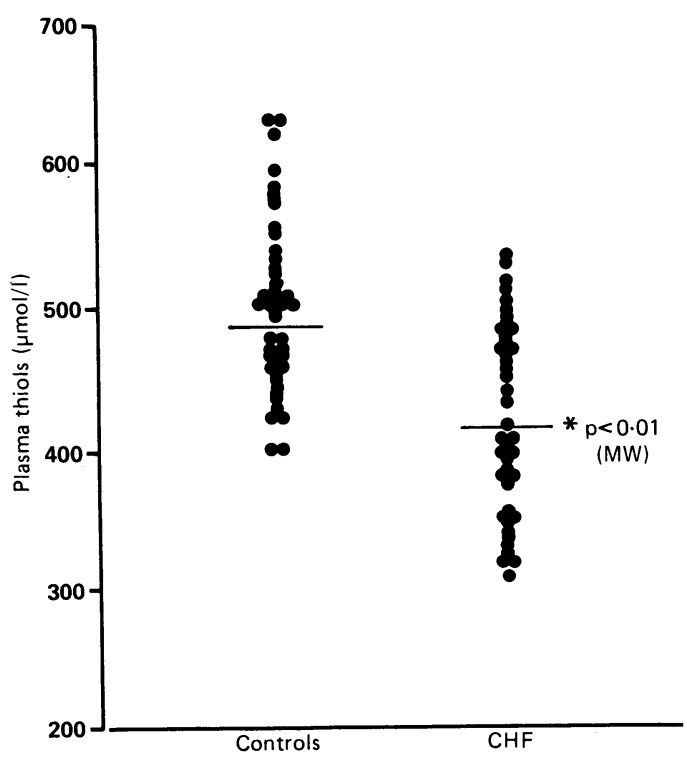

Figure 3 Plasma thiols in patients with congestive heart failure (CHF) and in controls. 
Figure 4 Plasma thiols and left ventricular ( $L V)$ ejection fraction in patients with congestive heart failure.

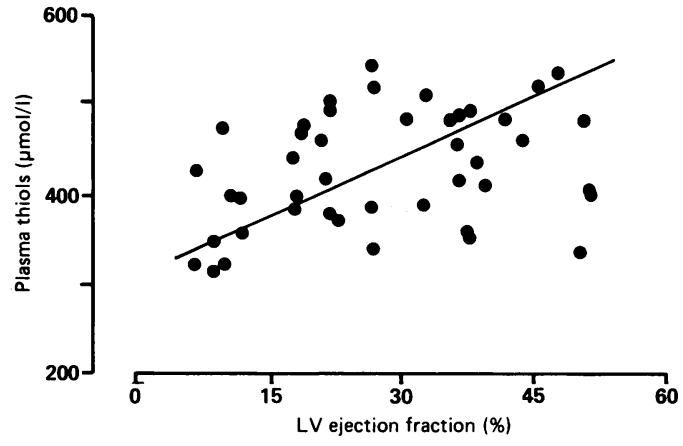

peroxides in the blood of patients with congestive heart failure. Moreover, there was a significant negative correlation with left ventricular ejection fraction, which is one of the best predictors of survival in congestive heart failure. ${ }^{12}$ Free radicals are unstable reactive chemical species produced during the course of normal metabolism that contain an unpaired electron. The half life of free radicals is measured in microseconds because they rapidly react with unoxidised adjacent molecules. They probably directly initiate tissue damage in several diseases where they are produced in excess. ${ }^{13-15}$ The reactivity of free radicals precludes their direct measurement in humans except by the most complex techniques. ${ }^{16}$ Their presence can be inferred, however, by their known effects, such as oxidation of polyunsaturated fatty acids. This is detected by the thiobarbituric acid test which detects the aldehyde products of peroxidised lipid and carbohydrates. ${ }^{10}$ Recent advances in assay methods suggest that high performance liquid chromatography is a more sensitive and specific assay for malondialdehyde-like material; however, this project was already under way before the high performance liquid chromatographic assay was validated in our laboratory. Nevertheless, we have no reason to believe that artefactual results have been produced and the abnormal concentrations of malondialdehyde-like material accord with the plasma thiol results. We found that freezing and storing the samples produced very variable results. By assaying the samples immediately after venesection we believe we reduced the variability seen with the spectrophotometric assay. The plasma thiol estimation measured albumin thiol which decreased in the presence of free radical activity owing to oxidation of the sulphydryl group to disulphides. ${ }^{17} 18$ The results of these tests support a theory that free radicals are involved in congestive heart failure.

We suggest therefore that oxygen free radicals are involved in the genesis and maintenance of congestive heart failure and there is theoretical support for this in published reports. Impairment of myocardial contractility or pressure or volume overload will all cause myocardial ischaemia, which in turn induces an increase in xanthine oxidase and xanthine and a decrease in the scavenging activity of superoxide dismutase and glutathione peroxidase ${ }^{19}$ Both these changes will lead to an increase in the generation of free radicals. The decrease in cellular $\mathrm{pH}$ during ischaemia will lead to formation of prostaglandins and leukotrienes. During their synthesis free radicals will be produced. ${ }^{8}$ Leukotriene $\mathrm{B}_{4}$ will further activate the polymorphonuclear leucocytes and increase secretion of neutrophil free radicals. ${ }^{20}$ The compensatory mechanism of an increase in catecholamines in congestive heart failure also increases free radicals. ${ }^{9}$ Free radicals themselves depress $\mathrm{Ca}^{2+}$ binding and uptake into sarcoplasmic reticulum ${ }^{21}$; this would lead to a decrease in cardiac contractility. Thus it is likely that free radicals actively promote the progression of congestive heart failure. Most of our patients had atherosclerosis underlying their congestive heart failure and the above mechanisms will contribute to the abnormalities detected. It would be of interest to study patients with congestive heart failure secondary to conditions such as a cardiomyopathy to see whether free radical products are also increased.

The development of treatments to reduce or prevent radical induced tissue injury may therefore be useful in congestive heart failure. There are two major classes of antioxidant: preventative antioxidants (for example, catalase, glutathione peroxidase, caeruloplasmin) and chain-breaking (radical-trapping) antioxidants (for example, vitamin $\mathrm{E}$, vitamin $C$, reduced glutathione). Certain compounds can be classified as both preventative and chainbreaking antioxidants because they have both functions (for example, superoxide dismutase, $\beta$ carotene). The angiotensin converting enzyme inhibitor, captopril, a well established drug used in the treatment of congestive heart failure, contains a sulphydryl group. Because one of the effects of sulphydryl-containing compounds is to scavenge free radicals, captopril may be a free radical scavenger. We have confirmed this both in vitro ${ }^{22}$ and in humans. ${ }^{23}$

We showed that patients with congestive heart failure have evidence of increased free radical activity that correlates with the left ventricular ejection fraction, a measure of heart function linked to prognosis. It is likely that congestive heart failure causes myocardial ischaemia with free radical production. Free radicals cause myocyte dysfunction, exacerbating the impaired cardiac function and a vicious cycle is set in motion with the net result that cardiac function steadily deteriorates. Some drugs used in the treatment of congestive heart failure may be effective scavengers and such treatments should be further investigated.

We thank Dr Norman Kennedy, Department of Nuclear Medicine, Ninewells Hospital for his help with the left ventricular ejection fraction measurements and $\mathrm{Dr} G \mathrm{McNeill}$ and Dr T Pringle for allowing us to study their patients. This Tringle for allowing us to study their patients. This study was supported by a grant to JJFB from the Britis Heart Foundation and the assays were carried out on equipmen purchased by a grant to JJFB from the Dundee University

1 Smith WM. Epidemiology of congestive heart failure. Am J Cardiol 1985;55:3A-8A.

2 Packer M. Sudden unexpected death in patients with congestive heart failure: a second frontier. Circulation congestive hea

3 McCord JM. Oxygen-derived free radicals in post ischaemic tissue injury. N Engl J Med 1985;312:159-63. 
4 Belch JJF, Chopra M, Hutchinson S, et al. Free radical pathology in chronic arterial disease. Free Radic Biol Med 1989;6:375-8.

5 Parmley WW. Pathophysiology of congestive heart failure. Am J Cardiol 1985;55:9A-14A.

6 Goodman J, Hochstein PL. Generation of free radicals and lipid peroxidation by redox cycling of Adriamycin and Dannomycin. Biochem Biophys Res Commun 1977;77: 797-803.

7 Prasad K, Kalra J, Bharadwaj B. Phagocytic activity in blood of dogs with chronic heart failure. Clin Invest Med 1987;10:1354-7.

8 Dzan VJ, Packer M, Lilly LS. Prostaglandins in severe congestive heart failure. Relation to activation of the renin-angiotensin system and hyponatremia. $N$ Engl J Med 1984;310:345-52.

9 Graham DG, Tiffany SM, Bell WR, Gutknecht WF. Autoxidation versus covalent binding of quinones as the idation versus covalent binding of quinones as the
mechanism of toxicity of dopamine, 6-hydroxydopamine and related compounds toward $\mathrm{C} 1300$ neuroblastoma cells and related compounds toward C1300 ne
in vitro. Mol Pharmacol 1978;14:644-53.

10 Aust SD. Lipid peroxidation. In: Greenwald RA, ed. Handbook of methods for oxygen radical research. Florida: CRC Press, 1988:203-7.

11 Ellman GE. Tissue sulphydryl groups. Arch Biochem Biophys 1969;82:70-7.

12 Hammermeister KE, De Ronen TA, Doge HT. Variables predictive of survival in patients with coronary disease. Circulation 1979;59:421-30.

13 Jennings PE, Jones AF, Florkowski CM, Lunec J, Barnett $\mathrm{AH}$. Increased diene conjugates in diabetic subjects with microangiopathy. Diab Med 1987;4:452-6.

14 Lunec J, Halloran SP, White AG, Dormandy TL. Free radical oxidation (peroxidation) products in serum and synovial fluid in rheumatoid arthritis. $J$ Rheumatol 1981 , 8:233-45

15 Belch JJF. Leukotrienes as mediators of inflammation In: Moll JMH, Sturrock RD, eds. Recent advances in rheumatology. London: Churchill Livingstone, 1986;4: 37-52

16 Rosen GM, Raukman EJ. Spin trapping of superoxide and hydroxyl radicals. In: Packer D, ed. Methods in enzymology. New York: Academic Press, 1984;105: 198-209.

17 Hall ND, Blake DR, Bacon PA. Serum sulphydryl levels as diagnostic and prognostic indicator in early rheumatoid arthritis. Ann Rheum Dis 1981;40:194-7.

18 Banford JC, Brown DH, Hazelton RA, McNeil CJ, Smith WE, Sturrock RD. Altered thiol status in patients with rheumatoid arthritis. Rheumatol Int 1982;2:107-11.

19 Guarnieri C, Flamigan F, Caldarera CM. Role of oxygen in the cellular damage induced by re-oxygenation of hypoxic the cellular damage induced by re-oxygenation
heart. J Mol Cell Cardiol 1980;12:797-808.

20 Mullane KM, Barst S, McGiff JC. Leukotrienes in myocardial ischaemia. In: Lefer AM, Gee MH, eds. Leukotrienes in cardiovascular and pulmonary function. New York: Alan

21 Hess ML, Okabe E, Kontos HA. Proton and free oxygen radical interaction with the calcium transport system of cardiac sarcoplasmic reticulum. J Mol Cell Cardiol 1981; 13:767-72.

22 Chopra M, Scott N, Smith WE, Bridges A, Belch JJF Captopril: a free radical scavenger. Br J Clin Pharmacol 1989;27:396-9

23 Chopra M, McMurray J, McLay J, et al. Oxidative damage in chronic heart failure. Protection by captopril through free radical scavenging. Adv Exp Med Biol 1991;264: $251-6$. 